\title{
The immune mechanism of intestinal tract Toll-like receptor in mediating EV71 virus type severe hand- foot-and-mouth disease and the MAPK pathway
}

\author{
LEI ZHU, WEIHUA LI, GONGJIAN QI, NA LIU, LIPING SHENG, LEI SHANG and BOXIANG QI
}

Department of Intensive Care Unit, Xuzhou Children's Hospital, Xuzhou, Jiangsu 221006, P.R. China

Received July 12, 2016; Accepted March 16, 2017

DOI: $10.3892 / e t m .2017 .4245$

\begin{abstract}
Immunological response is thought to play a crucial role in the development of a severe hand-foot-and-mouth disease (HFMD) infection in children, but the mechanisms remain largely unknown. This study was designed to help in elucidating the immunopathological pathways involved in the disease by quantifying Toll-like receptor (TLR) mRNAs, MAPK factors and cytokine levels in children experiencing the disease. A total of 86 enterovirus 71 (EV71)-infected HFMD children (49 with mild and 27 with severe disease), along with 30 healthy children were involved in the study. Peripheral vein blood samples were collected from each individual, and used to isolate peripheral blood mononuclear cells (PBMCs) for mRNA extraction and sera for measuring levels of tumor necrosis factor (TNF)- $\alpha$, interferon (IFN) $-\gamma$, interleukin (IL)-6 and IL-10. The average expression levels of TLR3, TLR4, TLR7 and TLR8 mRNA in PBMCs of children with severe HFMD were significantly higher than those in the other children, the lowest values were found in the healthy control group $(\mathrm{P}<0.05)$. The expression levels of TLR2 and TLR9 mRNA were not significantly different $(\mathrm{P}>0.05)$ among the groups. Additionally, the expression levels of TNF- $\alpha$, IFN- $\gamma$, IL- 6 and IL-10 in the serum of the children in the severe group were significantly higher than those in the other two groups, and the lowest values were again found in the control group $(\mathrm{P}<0.05)$. Pearson correlation analysis showed that the TLR3, TLR4, TLR7 and TLR8 mRNA levels in PBMCs were positively correlated with the TNF- $\alpha$, IFN- $\gamma$, IL- 6 and IL-10 levels in the serum $(\mathrm{P}<0.05)$. Furthermore, the expression levels of the ERK, JNK and p38 mRNA in PBMCs of children in the severe group were significantly higher than those in the other two groups, with the lowest values being in the control group $(\mathrm{P}<0.05)$. Pearson correlation analysis showed that the
\end{abstract}

Correspondence to: Dr Boxiang Qi, Department of Intensive Care Unit, Xuzhou Children's Hospital, 18 Sudi North Road, Xuzhou, Jiangsu 221006, P.R. China

E-mail: qi_boxiang1@163.com

Key words: severe hand-foot-and-mouth disease, enterovirus 71 type, Toll-like receptor, cytokine, MAPK signaling pathway
TLR3, TLR4, TLR7 and TLR8 mRNA levels in PBMCs were positively correlated with ERK, JNK and p38 mRNA levels $(\mathrm{P}<0.05)$. The results of our study seem to indicate that the high expression levels of TLR3, TLR4, TLR7 and TLR8 induced in severe EV71 HFMD regulate the expression of cytokines by MAPK signaling pathway and negatively affect the ability of the organism to resolve the infection. Further studies are needed to test the hypothesis that immuno-modulation would be an effective treatment approach in pediatric cases of severe HFMD.

\section{Introduction}

Hand-foot-and-mouth disease (HFMD) is an acute infectious disease with high incidence in preschool children, caused by a variety of enterovirus infections, and the clinical symptoms include fever, rash and sores on a plurality of sites of the hands, feet and mouth (1). Enterovirus 71 (EV71) and Coxsackie virus A16 are common etiological agents of HFMD, among which, EV71 can cause severe central nervous system damage and multiple-organ dysfunction and even become life threatening (2). EV71 infection can cause immune system dysfunction; T lymphocyte subsets can secrete a variety of inflammatory cytokines, which can worsen the disease symptoms (3).

Toll-like receptors (TLRs) are important pattern recognition molecules; those associated with the viral infection include TLR2, 3, 4, 7, 8 and 9 (4,5). After recognizing the virus, TLRs can activate multiple signaling pathways including the downstream MyD88-dependent and the MyD88-independent pathways, which regulate the expressions of a variety of inflammatory cytokines and are involved in inflammation and immune responses (6).

The present study was designed to analyze the immune mechanisms of intestinal TLRs and the MAPK signaling pathway after severe HFMD EV71 infection.

\section{Subjects and methods}

Subject information. A total of 86 children admitted to the Xuzhou Children's Hospital (Jiangsu, China) from January 2013 to January 2016 with a throat-swab-pathologic-examination-confirmed diagnosis of EV71 HFMD for the first time were contiguously enrolled in the study. A total 
Table I. Comparison of the expression levels of TLR mRNAs in PBMCs of subjects in the three groups.

\begin{tabular}{|c|c|c|c|c|c|c|}
\hline Groups & TLR2 & TLR3 & TLR4 & TLR7 & TLR8 & TLR9 \\
\hline Control & $1.00 \pm 0.16$ & $1.00 \pm 0.13$ & $1.00 \pm 0.09$ & $1.00 \pm 0.19$ & $1.00 \pm 0.15$ & $1.00 \pm 0.11$ \\
\hline Mild disease & $1.05 \pm 0.14$ & $1.89 \pm 0.26^{\mathrm{a}}$ & $1.91 \pm 0.24^{\mathrm{a}}$ & $1.77 \pm 0.20^{\mathrm{a}}$ & $1.58 \pm 0.23^{\mathrm{a}}$ & $1.08 \pm 0.15$ \\
\hline Severe disease & $1.09 \pm 0.12$ & $3.32 \pm 0.48^{\mathrm{a}, \mathrm{b}}$ & $3.88 \pm 0.59^{\mathrm{a}, \mathrm{b}}$ & $3.27 \pm 0.41^{\mathrm{a}, \mathrm{b}}$ & $3.06 \pm 0.52^{\mathrm{a}, \mathrm{b}}$ & $0.92 \pm 0.12$ \\
\hline
\end{tabular}

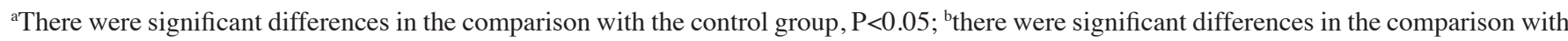
the mild disease group, $\mathrm{P}<0.05$. TLR, Toll-like receptor; PBMCs, peripheral blood mononuclear cells.

of 49 children had a mild disease and 27 children a severe form of the disease. The mild disease group included 29 boys and 20 girls, with a mean age of $4.5 \pm 0.6$ years; the severe disease group included 17 boys and 10 girls with a mean age of $4.9 \pm 0.8$ years. In addition, 30 healthy children were enrolled in a control group, including 18 boys and 12 girls with a mean age of $4.8 \pm 0.7$ years. Comparisons of general information between the three groups yielded no significant differences.

Study methods. Under fasting conditions, $6 \mathrm{ml}$ elbow venous blood samples were collected from each patient, and $2 \mathrm{ml}$ was used for separating peripheral blood mononuclear cells (PBMC) and testing for TLRs, $2 \mathrm{ml}$ for immune cytokine testing, and $2 \mathrm{ml}$ for MAPK signaling pathways testing.

Quantification of TLR and MAPK signaling molecule mRNA expression. Lymphocyte separation medium was added to peripheral blood samples and these were then centrifuged. The suspended PBMC were isolated in Eppendorf tubes. For RNA extraction, $1 \mathrm{ml}$ TRIzol lysis buffer (Invitrogen Life Technologies, Carlsbad, CA, USA) was added, and the closed tubes were inverted until well mixed, chloroform and isopropanol were then added before centrifugation. The pellets of RNA were washed twice with ethanol; and after the last concentration, purity and integrity were tested. Good quality RNA samples were inversely transcribed into cDNA with first-strand cDNA synthesis kit (Promega Corp., Madison, WI, USA), the experiments were carried out according to the fluorescence quantitative PCR kit instructions. Specific fragments from TLR2, 3, 4, 7, 8 and 9 as well as ERK, JNK and p38 mRNA were amplified, mRNA levels of each target gene were calculated normalizing against GAPDH RNA levels (internal reference).

Testing of cytokine levels in the serum. Peripheral blood samples were centrifuged at 2,000 x $\mathrm{g}$ for $20 \mathrm{~min}$, the upper serum layer was used in an enzyme-linked immunosorbent assay (ELISA). Briefly, an ELISA kit was used to test the tumor necrosis factor (TNF)- $\alpha$, interferon (IFN)- $\gamma$, interleukin (IL)-6 and IL-10 levels in the serum samples, the kit was purchased from the Sigma (St. Louis, MO, USA), and a microplate reader was purchased from Beijing Liuyi Instrument Factory (Beijing, China), and carried out according to the manufacturer's instructions.

Statistical analysis. The SPSS 20.0 software (IBM SPSS, Armonk, NY, USA) was used for data entry and processing.
Measurement data were expressed as mean \pm standard deviation, comparisons among groups were analyzed with a one-way ANOVA method, and pairwise comparisons were tested with an LSD-t-test method. Countable data were expressed as rates and the comparisons among groups were analyzed with a $\chi^{2}$ test. Finally, a Pearson correlation analysis of measurement data was done after a normality test. $\mathrm{P}<0.05$ was considered to indicate a statistically significant difference.

\section{Results}

Comparison of the expression levels of TLR $m R N A$ s among the groups. The average expression levels of TLR3, TLR4, TLR7 and TLR8 mRNAs in the isolated PBMCs of subjects in the three groups were significantly different $(\mathrm{P}<0.05)$, and those of TLR2 and TLR9 mRNAs were not $(\mathrm{P}>0.05)$. The average expression levels of TLR3, TLR4, TLR7 and TLR8 mRNAs in PBMCs of the severe disease group were significantly higher than those in the other two groups, with the lowest values being found in the control group $(\mathrm{P}<0.05$; Table I).

Comparison of expression levels of cytokines in the serum. The average expression levels of TNF- $\alpha$, IFN- $\gamma$, IL- 6 and IL-10 in the serum of subjects in the three groups were significantly different. Average expression levels of TNF- $\alpha$, IFN- $\gamma$, IL-6 and IL-10 in the serum of the severe disease group were higher than those in the other two groups, the lowest values were found in the control group $(\mathrm{P}<0.05$; Table II).

Correlation between TLR levels in PBMCs and cytokine levels in serum. Pearson correlation analysis showed that the TLR3, TLR4, TLR7 and TLR8 mRNA levels in PBMCs were positively correlated with TNF- $\alpha$, IFN- $\gamma$, IL-6 and IL-10 levels in the serum $(\mathrm{P}<0.05$; Table III).

Comparison of expression levels of MAPK signaling molecules in PBMCs. The average expression levels of ERK, JNK and p38 mRNA in PBMCs of subjects in the three groups were significantly different $(\mathrm{P}<0.05)$. Those of ERK, JNK and p38 mRNA in PBMCs of the children in the severe disease group were significantly higher than those in the other two groups, the lowest values were found in the control group $(\mathrm{P}<0.05$; Table IV).

Correlation between TLR in PBMCs and MAPK mRNA levels. Pearson correlation analysis showed that the TLR3, TLR4, TLR7 and TLR8 mRNA levels in PBMCs were positively 
Table II. Comparison of expression levels of cytokines in serum of subjects in the three groups.

\begin{tabular}{lcccc}
\hline Groups & TNF- $\alpha, \mathrm{ng} / \mathrm{ml}$ & IFN- $\gamma, \mathrm{pg} / \mathrm{ml}$ & IL-6, ng/ml & IL-10, pg/ml \\
\hline Control & $11.48 \pm 1.86$ & $7.68 \pm 0.92$ & $23.58 \pm 5.86$ & $9.14 \pm 0.15$ \\
Mild disease & $18.59 \pm 2.49^{\mathrm{a}}$ & $18.51 \pm 2.64^{\mathrm{a}}$ & $47.64 \pm 7.12^{\mathrm{a}}$ & $17.79 \pm 2.52^{\mathrm{a}}$ \\
Severe disease & $55.75 \pm 8.64^{\mathrm{a}, \mathrm{b}}$ & $30.24 \pm 5.42^{\mathrm{a}, \mathrm{b}}$ & $142.86 \pm 25.38^{\mathrm{a}, \mathrm{b}}$ & $28.58 \pm 4.82^{\mathrm{a}, \mathrm{b}}$
\end{tabular}

${ }^{a}$ There were significant differences in the comparison with the control group, $\mathrm{P}<0.05$; ${ }^{\text {there }}$ were significant differences in the comparison with the mild disease group, $\mathrm{P}<0.05$. TNF, tumor necrosis factor; IFN, interferon; IL, interleukin.

Table III. Correlation between TLR mRNA levels in PBMCs and cytokine levels in serum.

\begin{tabular}{|c|c|c|c|c|c|c|c|c|}
\hline & \multicolumn{2}{|c|}{ TLR3 } & \multicolumn{2}{|c|}{ TLR4 } & \multicolumn{2}{|c|}{ TLR7 } & \multicolumn{2}{|c|}{ TLR8 } \\
\hline & t-test & $\mathrm{P}$-value & t-test & P-value & t-test & P-value & t-test & P-value \\
\hline TNF- $\alpha$ & 0.284 & 0.035 & 0.301 & 0.030 & 0.346 & 0.023 & 0.348 & 0.022 \\
\hline IFN- $\gamma$ & 0.315 & 0.032 & 0.281 & 0.026 & 0.348 & 0.022 & 0.310 & 0.024 \\
\hline IL-6 & 0.302 & 0.033 & 0.268 & 0.028 & 0.328 & 0.025 & 0.367 & 0.018 \\
\hline IL-10 & 0.325 & 0.030 & 0.296 & 0.025 & 0.303 & 0.028 & 0.349 & 0.015 \\
\hline
\end{tabular}

TLR, Toll-like receptor; PBMCs, peripheral blood mononuclear cells; TNF, tumor necrosis factor; IFN, interferon; IL, interleukin.

Table IV. Comparison of expression levels of MAPK signaling molecules in PBMCs of subjects in the three groups.

\begin{tabular}{llll}
\hline Groups & ERK & JNK & p38 \\
\hline Control & $1.00 \pm 0.18$ & $1.00 \pm 0.14$ & $1.00 \pm 0.17$ \\
Mild disease & $1.83 \pm 0.25^{\mathrm{a}}$ & $1.59 \pm 0.21^{\mathrm{a}}$ & $1.77 \pm 0.27^{\mathrm{a}}$ \\
Severe disease & $3.52 \pm 0.58^{\mathrm{a}, \mathrm{b}}$ & $3.28 \pm 0.52^{\mathrm{a}, \mathrm{b}}$ & $2.89 \pm 0.39^{\mathrm{a}, \mathrm{b}}$ \\
\hline
\end{tabular}

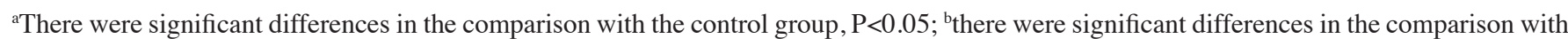
the mild disease group, $\mathrm{P}<0.05$. PBMCs, peripheral blood mononuclear cells.

Table V. Correlation between TLR mRNA levels in PBMCs and MAPK levels.

\begin{tabular}{|c|c|c|c|c|c|c|c|c|}
\hline & \multicolumn{2}{|c|}{ TLR3 } & \multicolumn{2}{|c|}{ TLR4 } & \multicolumn{2}{|c|}{ TLR7 } & \multicolumn{2}{|c|}{ TLR8 } \\
\hline & t-test & P-value & t-test & P-value & t-test & P-value & t-test & P-value \\
\hline ERK & 0.485 & 0.016 & 0.512 & 0.014 & 0.482 & 0.016 & 0.509 & 0.016 \\
\hline JNK & 0.483 & 0.017 & 0.472 & 0.018 & 0.518 & 0.013 & 0.542 & 0.012 \\
\hline p38 & 0.527 & 0.012 & 0.542 & 0.010 & 0.509 & 0.015 & 0.567 & 0.009 \\
\hline
\end{tabular}

TLR, Toll-like receptor; PBMCs, peripheral blood mononuclear cells.

correlated with ERK, JNK and p38 mRNA levels $(\mathrm{P}<0.05$; Table V).

\section{Discussion}

The immune function of organisms are activated after EV71 infection and secreted cytokine levels by a variety of immune cells increase. On the one hand, these changes are involved in the organism immune protection; on the other hand they can also cause immunopathological injury to the organism. The balance of Th1/Th2 cells is an important mechanism regulating immune function. Th1 cells can synthesize TNF- $\alpha$, IFN- $\gamma$ and IL-6, which mainly mediate the cell immune response and play a major role in the antiviral process (7). Th2 
cells can synthesize IL-10, inhibit excessive activation of Th1 cells, promote the proliferation of B cells and mainly mediate the humoral immune response (8). Studies have confirmed that the functions of Th1 and Th 2 cells in children with HFMD are significantly altered and the synthesis and secretion of cytokines are increased $(9,10)$. In our study, it was found that the expression levels of TNF- $\alpha$, IFN- $\gamma$, IL- 6 and IL-10 in the serum of children in the severe disease group were significantly higher than those in the mild disease and the control groups. This is consistent with the cited previous study results. The above cytokines synthesized by Th1 and Th2 cells are not only involved in the organism's protective immune response, they simultaneously promote an inflammatory reaction that can cause adverse reactions. In particular, cytokines synthesized by Th1 cells can cause a systemic inflammatory response syndrome (11) when attempting to clear the virus infections, that is why a Th2 cell response antagonizes the excessive inflammatory response in vivo, acting as the organism's own compensatory defense mechanism (12). When the cytokines synthesized by Th1 and Th2 in pediatric patients with HFMD are increased significantly, they cause a strong traumatic response syndrome (13).

Currently, the changes in levels of Th1 and Th2 secretions in pediatric patients with HFMD, and the mechanisms involved in the regulation of the resulting inflammatory cytokine syntheses are not clear. TLRs are considered the most important pattern recognition molecules in vivo, after a viral infection, a conformational change occurs in them, which leads to activation of a signal transduction through the MyD88-dependent and MyD88-independent pathways. An inflammatory response ensues with diverse cytokines regulating the expression of other inflammatory factors. The present study found that the expression levels of the TLR3, TLR4, TLR7 and TLR8 mRNAs in PBMCs of children in the severe disease group were significantly higher than those in the mild disease and the control groups. And that those levels were positively correlated with TNF- $\alpha$, IFN- $\gamma$, IL-6 and IL-10 levels in the serum. It is therefore possible that TLRs regulate the Th1 and Th2 cytokine production in the child patients with HFMD and help regulate the immune response of HFMD $(14,15)$.

Following TLR activation a cascade of events occurs leading especially to MAPK activation. Then, the signaling pathways mediated by the three signal molecules ERK, JNK and p38 activate c-Jun and c-Fos in the nucleus of lymphocytes and start the transcription of a variety of inflammatory factors $(16,17)$. The present study found that the expression levels of ERK, JNK and p38 mRNA in PBMCs of pediatric patients, in the severe disease group, were significantly higher than those in the mild disease group and the control group, and, that the TLR3, TLR4, TLR7 and TLR8 mRNA levels in PBMCs were positively correlated with ERK, JNK and p38 mRNA levels. From these results, it looks as though the high expression levels of TLR3, TLR4, TLR7 and TLR8 in severe EV71 HFMD regulate cytokine expression by the MAPK pathway and the resulting exaggerated response causes a severe and dangerous syndrome. It is possible that an immunological intervention, downregulating the expression of TLRs, may be a new target in the treatment of severe HFMD.

\section{References}

1. Long DL, Zhu SY, Li CZ, Chen CY, Du WT and Wang X: Late-onset nail changes associated with hand, foot, and mouth disease: A clinical analysis of 56 cases. Pediatr Dermatol 33: 424-428, 2016.

2. Kim C, Kang H, Kim DE, Song JH, Choi M, Kang M, Lee K, Kim HS, Shin JS, Jeong H, et al: Antiviral activity of micafungin against enterovirus 71. Virol J 13: 99, 2016.

3. Li S, Cai C, Feng J, Li X, Wang Y, Yang J and Chen Z: Peripheral $\mathrm{T}$ lymphocyte subset imbalances in children with enterovirus 71-induced hand, foot and mouth disease. Virus Res 180: 84-91, 2014.

4. Yang J, Yang C, Guo N, Zhu K, Luo K, Zhang N, Zhao H, Cui Y, Chen L, Wang H, et al: Type I interferons triggered through the Toll-like receptor 3-TRIF pathway control coxsackievirus A16 infection in young mice. J Virol 89: 10860-10867, 2015.

5. Hsiao HB, Chou AH, Lin SI, Chen IH, Lien SP, Liu CC, Chong P and Liu SJ: Toll-like receptor 9-mediated protection of enterovirus 71 infection in mice is due to the release of danger-associated molecular patterns. J Virol 88: 11658-11670, 2014.

6. Chi J, He Y, Yu G and Lei L: Role of Toll-like receptor 7 in the production of inflammatory cytokines in EV-A71-infected human Jurkat T cells. Zhonghua Yu Fang Yi Xue Za Zhi 50: 266-269, 2016 (In Chinese).

7. Dang S, Gao N, Li Y, Li M, Wang X, Jia X, Zhai S, Zhang X, Liu J, Deng H, et al: Dominant CD4-dependent RNA-dependent RNA polymerase-specific T-cell responses in children acutely infected with human enterovirus 71 and healthy adult controls. Immunology 142: 89-100, 2014.

8. Wei R, Xu L, Zhang N, Zhu K, Yang J, Yang C, Deng C, Zhu Z, De Groot AS, Altmeyer R, et al: Elevated antigen-specific Th2 type response is associated with the poor prognosis of hand, foot and mouth disease. Virus Res 177: 62-65, 2013.

9. Tan S, Tan X, Sun X, Lu G, Chen CC, Yan J, Liu J, Xu W and Gao GF: VP2 dominated $\mathrm{CD}^{+} \mathrm{T}$ cell responses against enterovirus 71 and cross-reactivity against coxsackievirus A16 and polioviruses in a healthy population. J Immunol 191: 1637-1647, 2013.

10. Liu L, Zhang Y, Wang J, Zhao H, Jiang L, Che Y, Shi H, Li R, Mo Z, Huang T, et al: Study of the integrated immune response induced by an inactivated EV71 vaccine. PLoS One 8: e54451, 2013.

11. Wei R, Yang C, Zeng M, Terry F, Zhu K, Yang C, Altmeyer R, Martin W, De Groot AS and Leng Q: A dominant EV71-specific $\mathrm{CD}^{+} \mathrm{T}$ cell epitope is highly conserved among human enteroviruses. PLoS One 7: e51957, 2012.

12. Liang Y, Zhou X, Yang E, Pu J, Che Y, Wang J, Ma N, Liu L, Ding D, Tang D, et al: Analysis of the Th1/Th2 reaction in the immune response induced by EV71 inactivated vaccine in neonatal rhesus monkeys. J Clin Immunol 32: 1048-1058, 2012.

13. Dong C, Liu L, Zhao H, Wang J, Liao Y, Zhang X, Na R, Liang Y, Wang $\mathrm{L}$ and $\mathrm{Li}$ Q: Immunoprotection elicited by an enterovirus type 71 experimental inactivated vaccine in mice and rhesus monkeys. Vaccine 29: 6269-6275, 2011.

14. Lei X, Sun Z, Liu X, Jin Q, He B and Wang J: Cleavage of the adaptor protein TRIF by enterovirus 71 3C inhibits antiviral responses mediated by Toll-like receptor 3. J Virol 85: 8811-8818, 2011.

15. Zhang J, Wang J, Pang L, Xie G, Welte T, Saxena V, Wicker J, Mann B, Soong L, Barrett A, et al: The co-stimulatory effects of MyD88-dependent Toll-like receptor signaling on activation of murine $\gamma \delta$ T cells. PLoS One 9: e108156, 2014.

16. Zou J and Shankar N: Roles of TLR/MyD88/MAPK/NF- $\kappa B$ signaling pathways in the regulation of phagocytosis and proinflammatory cytokine expression in response to $E$. faecalis infection. PLoS One 10: e0136947, 2015.

17. Choi HG, Kim WS, Back YW, Kim H, Kwon KW, Kim JS, Shin SJ and Kim HJ: Mycobacterium tuberculosis RpfE promotes simultaneous Th1- and Th17-type T-cell immunity via TLR4-dependent maturation of dendritic cells. Eur J Immunol 45: 1957-1971, 2015. 